\title{
Robot-Assisted Tower Construction-A Method to Study the Impact of a Robot's Allocation Behavior on Interpersonal Dynamics and Collaboration in Groups
}

\author{
MALTE F. JUNG and DOMINIC DIFRANZO, Cornell University \\ SOLACE SHEN, Robinhood \\ BRETT STOLL and HOUSTON CLAURE, Cornell University \\ AUSTIN LAWRENCE, RoBotany
}

\begin{abstract}
Research on human-robot collaboration or human-robot teaming, has focused predominantly on understanding and enabling collaboration between a single robot and a single human. Extending human-robot collaboration research beyond the dyad, raises novel questions about how a robot should allocate resources among group members and about what the consequences of such allocation are for a group's social dynamics and outcomes. Methodological advances are needed to answer these questions allow researchers to collect data about a robot's impact not only on interactions with the robot but also on interactions of people with each other. This paper presents Robot Assisted Tower Construction, a novel task that allows researchers to examine the impact of a robot's allocation behavior on the dynamics of a group or team collaborating on a task. By focusing on the question of whether and how a robot's allocation of resources (wooden blocks required for a building task) affects collaboration dynamics and outcomes, a case is provided of how this task can be applied in a laboratory study with 124 participants to collect data about human robot collaboration that involves a group of people. We highlight the kinds of insights the task can yield and how it can be adapted to various human robot collaboration contexts.
\end{abstract}

CCS Concepts: $\bullet$ Human-centered computing; • General and reference $\rightarrow$ Cross-computing tools and techniques; Empirical studies; • Applied computing $\rightarrow$ Psychology;

Additional Key Words and Phrases: Human-robot collaboration, interpersonal dynamics, human-robot teaming, human-robot interaction, research method, groups, and teams

\section{ACM Reference format:}

Malte F. Jung, Dominic Difranzo, Solace Shen, Brett Stoll, Houston Claure, and Austin Lawrence. 2020. RobotAssisted Tower Construction-A Method to Study the Impact of a Robot's Allocation Behavior on Interpersonal Dynamics and Collaboration in Groups. ACM Trans. Hum.-Robot Interact. 10, 1, Article 2 (October 2020), 23 pages.

https://doi.org/10.1145/3394287

The work presented in this article is supported by the National Science Foundation under grants \#1422484 and \#1139161. Authors' addresses: M. F. Jung, D. DiFranzo, B. Stoll, and H. Claure, Cornell University, Ithaca, NY, 14850; emails: \{mjung, djd274, bas364, hbc35\}@cornell.edu; S. Shen, Robinhood, Menlo Park, CA, 94025; email: solaceshen@gmail.com; A. Lawrence, RoBotany, Pittsburgh, PA, 15213; email: ablarry91@gmail.com.

Permission to make digital or hard copies of all or part of this work for personal or classroom use is granted without fee provided that copies are not made or distributed for profit or commercial advantage and that copies bear this notice and the full citation on the first page. Copyrights for components of this work owned by others than the author(s) must be honored. Abstracting with credit is permitted. To copy otherwise, or republish, to post on servers or to redistribute to lists, requires prior specific permission and/or a fee. Request permissions from permissions@acm.org.

(C) 2020 Copyright held by the owner/author(s). Publication rights licensed to ACM.

2573-9522/2020/10-ART2 \$15.00

https://doi.org/10.1145/3394287 


\section{INTRODUCTION}

Advancements in safe human-robot interaction (see Lasota et al. [2017] and Bauer et al. [2008] for reviews) have led to a steady increase in research on human-robot collaboration and teaming [Ajoudani et al. 2018] over recent years. However, to date, this work has focused almost exclusively on a single robot collaborating with a single human. We have little understanding about human robot collaboration and interaction with groups of people and a robot's impact on group dynamics and outcomes. Building such understanding is important since people rarely work in isolation. Work almost always involves groups or teams of people to some degree [Kozlowski and Bell 2003; Wuchty et al. 2007], even in manufacturing (e.g., see Banker et al. [1996], Durand et al. [1999], Vallas [2003], and Ohno [1988]), where collaborative robots are increasingly envisioned or employed.

Expanding human-robot collaboration and interaction toward groups and teams of people raises a range of novel questions that cannot be answered by current human-robot collaboration approaches and theory. For example, techniques for turn-taking do not easily scale from dyads to groups (e.g., see Matsusaka et al. [2001] and Matsuyama et al. [2015]). Further, scaling humanrobot collaboration to groups and teams raises questions about a robot's influence not only about the interactions that people engage in with the robot but also about the interactions that people engage in with each other. Understanding the latter is important since many studies have shown that the performance of groups and teams highly depends on a group's interaction and dynamics (e.g., see Jung [2016], Jung et al. [2012], Woolley et al. [2015], McGrath [1991], Hackman and Morris [1975], and McGrath [1984]), and recent studies have shown that robots have the power to shape those dynamics in complex ways (e.g., see Mutlu et al. [2009], Strohkorb Sebo et al. [2018], Correia et al. [2018], and Tennent et al. [2019]). To build systems that can successfully collaborate with groups of people, it is important to develop a systematic understanding about a robot's influence on group dynamics.

One of the most consequential differences between human robot collaboration and interaction with one person versus a group of people is that, in the latter, the robot has to make decisions about the the allocation of resources, e.g., whom it is paying attention to, or whom it is assisting or collaborating with at any instance. Making such decisions results in resource allocation behavior, i.e., the outward, observable behavior in which a robot acts upon its resource allocation decision [Langholtz et al. 2002]. Studies have shown that people are uniquely attuned to the allocation of resources [Brosnan and de Waal 2014; Lee 2018]. The mere allocation of a robot's gaze among members of a group has shown to influence the roles people take on within a group [Mutlu et al. 2009]. It is not clear how a robot should make such decisions and what the consequences of a robot's resource allocation behaviors are for a group's dynamics and task performance.

We present Robot Assisted Tower Construction, a novel human-robot collaboration task that allows researchers to collect data about the impact of a robot's allocation behavior on human-robot collaboration and interaction with groups. Our task extends basic human-robot collaboration approaches (e.g., as in Hayes and Scassellati [2014, 2015]) by adding one or more human collaborators (Figure 1). We provide a case of how this task can be applied in research by focusing on the question of how the unequal allocation of resources (wooden blocks required for a collaborative building task involving two humans) affects interpersonal collaboration dynamics and outcomes.

The article makes three contributions to the human-robot interaction (HRI) literature: First, we contribute a task that enables researchers to explore questions that arise when we extend human-robot collaboration to groups of people. The task allows researchers to not only study how a robot's allocation behavior impacts interactions of group members with the robot, but also to study how a robot's allocation behavior affects people's interactions with each other (Figure 2). 


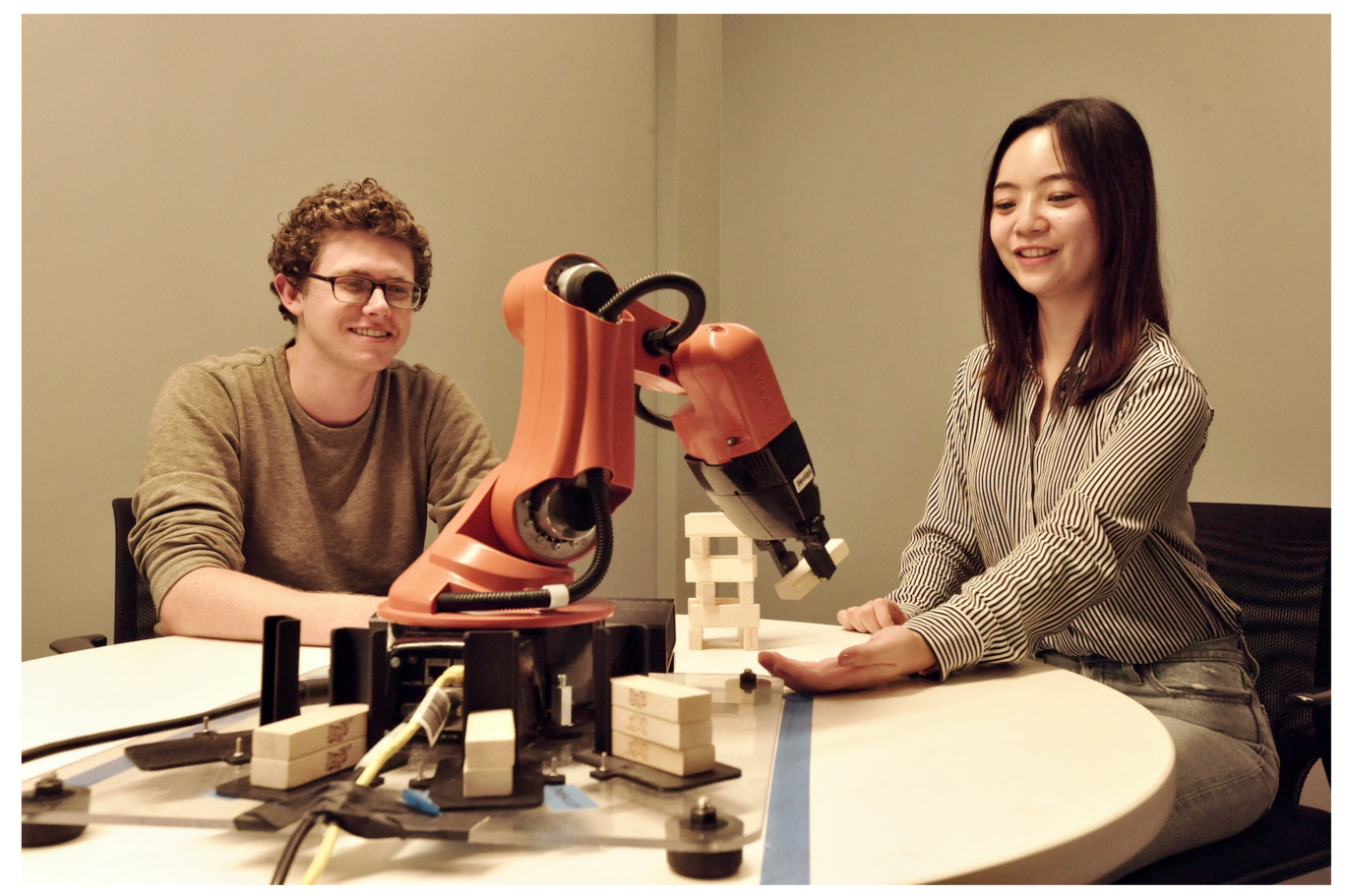

Fig. 1. Two participants working as a team in completing a construction task in collaboration with a robot.

Our work constitutes a methodological contribution as it directly informs how research is carried out in HRI [Wobbrock 2012; Wobbrock and Kientz 2016] by enabling researchers to collect data about human robot collaborations that involve more than one human. Efforts to scale human-robot collaboration to groups depend on understanding about how robots influence social dynamics in groups. Our task offers a platform to build such understanding. Second, we demonstrate the utility of our task through a case study that explores the impact of unequal resource allocation on interpersonal dynamics and task performance and by highlighting a range of other possible use cases. Our case study provides evidence that a robot distributing resources unequally between two human collaborators affects interpersonal dynamics in systematic ways. Third, our task offers a context to collect data on social dynamics in human robot collaboration without requiring a robot that is designed with explicit social signaling capabilities (e.g., gaze, speech, or color displays). While previous research has shown that people can make sense of any robot in social terms and, thus, recognize any machine as a social actor (e.g., see Forlizzi and DiSalvo [2006] and Reeves and Nass [1996]), our task and case study extend this work by demonstrating that non-humanoid robots can also powerfully influence inter-human social dynamics.

\section{STUDYING HUMAN-ROBOT INTERACTION AND COLLABORATION IN GROUPS AND TEAMS}

To position our work within existing HRI research, we provide an overview of existing research on human-robot interaction in group and team settings. This review does not attempt to provide a comprehensive overview but rather aims at highlighting current trends and perspectives in HRI research on robots in group contexts. 


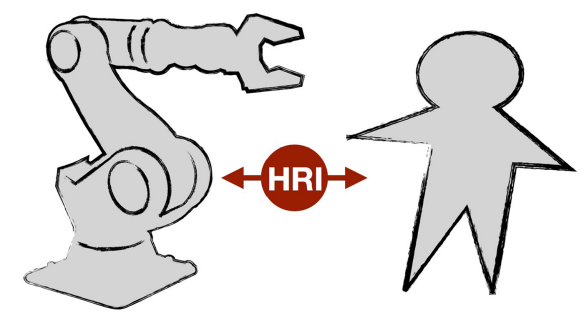

\section{ONE-TO-ONE}

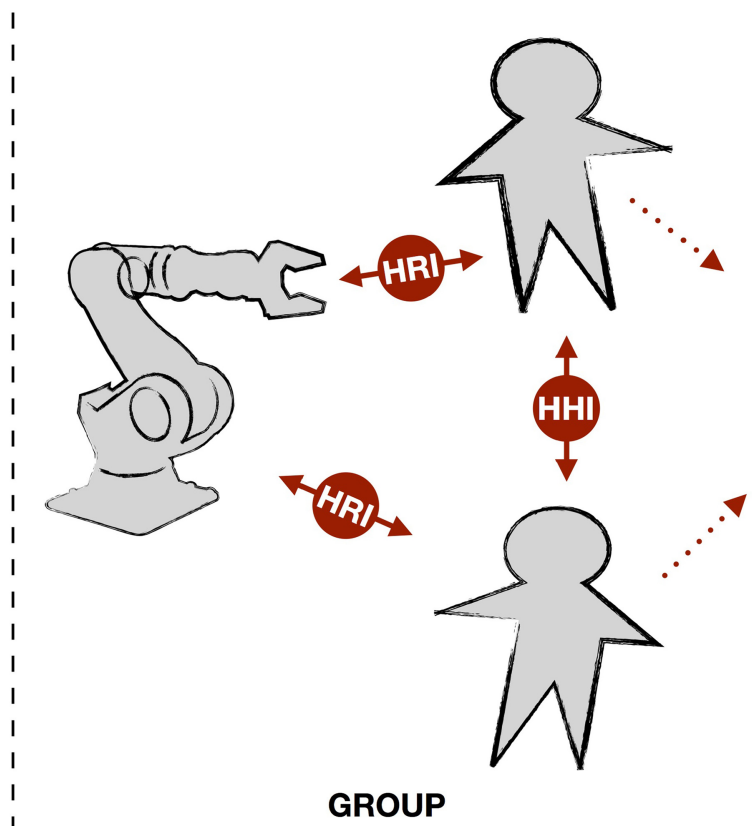

Fig. 2. Comparison between standard dyadic one-human-one-robot interaction/collaboration paradigm and human-robot interaction (HRI) with groups of people. Building understanding about human-robot interaction and collaboration in groups and teams requires consideration not only of HRI but also of human-human interactions ( $\mathrm{HHI}$ ). Thus, human-robot collaboration in groups and teams is different from dyadic collaboration not only because of the larger number of people but also because interpersonal dynamics are introduced.

\subsection{Human-Robot Collaboration and Teaming}

A growing amount of work on human-robot teaming has developed important insights toward understanding and enabling teamwork with embodied autonomous robots. Human-robot collaboration, or teaming, refers to collaborative partnerships between humans and robots in completing tasks and typically focuses on coordinating close, seamless joint activities between a single human and a single robot (for a recent review, see Ajoudani et al. [2018]). A central premise behind work to enable human-robot collaboration is that the combination of a robot's unique capabilities with those of humans opens possibilities that are out of reach for robots or humans each working in isolation [Shah 2017]. This involves a variety of behavioral and technical solutions for anticipating, planning, and executing partner actions (e.g., see Hayes and Scassellati [2016], Hoffman and Breazeal [2007], and Shah et al. [2011]), generating interpretable behavior (e.g., see Dragan et al. [2013] and Knepper et al. [2017]), developing novel methods (e.g., crowdsourcing human behavior for model generation [Breazeal et al. 2013]), as well as studying effects of such collaborative partnerships on people (e.g., Hinds et al. [2004]). Several studies have also explored how human-robot teamwork can be improved through a robot's social behavior. For example, Scheutz et al. [2006] found that team performance in a collaborative search task could be improved through emotional changes in the robot's voice tone, and several studies explored the important role of gaze in improving human-robot collaboration by studying handovers (e.g., see Admoni et al. [2014] and Moon et al. [2014]). Another study of a teamwork scenario in which one human participant interacted with two autonomous robots found that robots can improve teamwork through subtle displays of positive engagement and interest alone [Jung et al. 2013]. While this work has been highly 
impactful in providing behavioral and computational insight into understanding and improving collaborative partnerships between a single human and a single robot, it does not, besides a few exceptions (e.g., see Jung et al. [2015], Strohkorb Sebo et al. [2018], and You and Robert [2017]), speak to contexts in which robots collaborate with more than a single person.

\subsection{Robots in Work Contexts}

Studies of autonomous embodied robots and their effects on people in work contexts began as early as the 80 s, soon after industrial robots saw increased use in manufacturing. For example, work by Linda Argote and colleagues (e.g., see Argote and Goodman [1985] and Argote et al. [1983]) demonstrated both advantages (reduced fatigue) and disadvantages (overabundance of human downtime) from the perspective of human workers. While these robots had to be employed in areas caged off from humans, recent advances in safe HRI have made it possible for humans to interact closely with robots without fear of, harm, injury, or death (see Lasota et al. [2017] for a review), and robots now support work in close contact with humans in a wide range of areas. For example, a recent study by Sauppe and Mutlu [2015] showed that a robot's treatment as a social entity extends to industrial settings in which robots closely work with humans on manufacturing tasks. Research on hospital delivery robots has demonstrated that how people respond to a robot depends on patient needs, work practices within a given unit [Mutlu and Forlizzi 2008], and job roles [Siino and Hinds 2005]. The introduction of robots can alter work practices and occupations. In a study of two pharmacy delivery robots in the UK, Barrett et al. [2012] found that pharmacy technicians expanded their role by tending to the robot, whereas pharmacy assistants lost control over their work tasks, which were increasingly dictated by the demands of the robot and the technicians. Studies of science teams operating remote rovers also tell us that working with a remote autonomous robot can alter fundamental aspects of scientists' practices [Vertesi 2015] and that increased autonomy can lead to questions about how to interpret a robot's activities [Stubbs et al. 2007]. In sum, studies of robots in work contexts have provided vital information about how robots change the way that people work, how people from different professions interact with robots, and how activities surrounding interactions with robots affect peoples' behavior and attitudes toward robots. However, we have little understanding about the mechanisms through which robots influence interpersonal dynamics in teams, despite decades of research on teams that establishes such dynamics as crucial for the short- and long-term performance of teams.

\subsection{Robots in Group and Team Settings}

A growing number of studies have demonstrated that embodied autonomous robots can affect their social environment beyond the person interacting with them. Such influence can play out in multiple ways. We know that robots can take the role of a mediator and actively influence how two or more people relate to or interact with one another. Early studies of robots used in autism therapy, for example, show that robots can shape how children socially interact with others (for a review, see Scassellati et al. [2012]). Further, Hoffman and colleagues [2015] presented a robot designed to diffuse tension in marital conflict through nonverbal fear expressions [Hoffman et al. 2015] and a recent study by Shen et al. [2018] demonstrated the effectiveness of a robot in helping young children resolve interpersonal resource conflicts. Expanding the focus from dyads to larger groups, Short and colleagues [2017] showed that intergenerational interactions between older adults and their families could be shaped through a robot's behavior. Another study by Correia and colleagues [2018] showed that a group's shared emotional state during a card playing game could be influenced through a robot's self or group-oriented emotional statements. Several other studies have explored how the way a robot moves in a group affects coordination dynamics in groups engaged in group dance (e.g., see Iqbal et al. [2016] and Iqbal and Riek [2017]). Studies have 
also begun to explore how groups of people are affected through subtle, often non-verbal behavior of a robot rather than through explicit moderation attempts. For example, Mutlu and colleagues [2009] studied groups of museum visitors and demonstrated that even subtle, nonverbal gaze cues are sufficient to impact entire groups by shaping social roles. A study by Oliveira and colleagues [2018] showed that the amount of pro-social behavior a group of people exhibited while playing a game was dependent on the robot's displayed goal orientation as friend or foe. Finally, a study by Vazquez and colleagues [2017] showed that a robot's social orientation within a group of people affected social gaze patterns toward the robot.

Even robots that are not intentionally designed with a moderating role can shape interpersonal interactions in groups, for example, by shaping norms of social conduct through their behavior. Lee and colleagues documented "ripple effects" of a robot's behavior as it influenced how people interacted with each other and as it influenced the shared norms that people developed regarding appropriate ways of interacting with the robot [Lee et al. 2012]. Longitudinal studies of the Robovie robot in shopping malls also show that people tend to approach a robot in groups and are more likely to act on the robot's advice about what stores to visit if they feel they have a relationship with it [Kanda et al. 2010]. There is also evidence that a robot's mere presence can affect how people interact with each other [Dole 2017; Riether et al. 2012]. Dole [2017], for example, showed that participants looked at a nutritionist more and perceived her as less warm when in the presence of a physically embodied social robot as compared to a tablet computer or a nonsocial robot.

These studies provide strong evidence that robots can influence people beyond the person interacting directly with the robot and thus shape how people behave and interact with others, the roles that people assume in groups, and even the norms that groups develop. However, despite this evidence for a robot's impact beyond the individual, most studies have been focusing on groups in family and play contexts (such as supporting family communication, or playing a game or dancing together) rather than on groups or teams in work or teamwork contexts.

\subsection{Summary}

Taken as a whole, these studies reflect a growing interest in building understanding about a robot's influence on groups and their dynamics, and about how robots can be deliberately designed with such group influence in mind. This growing interest also highlights the importance of the methods HRI researchers have at their disposal when studying robots in groups and teams. For example, Oliveria and colleagues [2019] argued for the need to develop validated metrics that allow researchers to build deeper understanding about human-robot group dynamics.

Besides developing appropriate metrics to assess human-robot collaboration and interaction in group and team settings, it is important to develop tasks that elicit human-robot group interactions. For example, tasks such as the desert survival problem [Lafferty et al. 1974] or the hidden profile tasks [Stasser and Titus 1985] have been crucial in eliciting group interactions that allowed researchers to build fundamental understanding about group decision-making and information sharing, respectively. To understand HRI and collaboration in groups and teams, it is important to develop tasks that elicit such collaborative interactions with robots.

While current HRI research on interaction and collaboration in groups reflects a wide range of tasks, these tasks share several characteristics that limit the kinds of insights researchers can gain about human-robot collaboration and interaction in groups. First, existing tasks are often highly specific to the phenomenon under study (e.g., mistake admissions [Strohkorb Sebo et al. 2018]) or reactions to a hostile remark by a group member [Jung et al. 2015] and rarely set up more general collaborative situations. Second, most current group studies employ tasks that rely on tablet or screen-based games (e.g., see Strohkorb Sebo et al. [2018], Correia et al. [2018], and Oliveira et al. [2018]). While this makes it easier for a robot to assess the state of the tasks, it constrains 
interactions to mostly verbal and non-physical interactions. Finally, current tasks have relied almost exclusively on robots with human-like physical features and human-like interaction modalities [Tennent et al. 2019]. These tasks are not designed to accommodate non-anthropomorphic robots such as industrial robot arm even though such types of robots are the ones that are most widely deployed. Given these limitations, the goal of our article is to introduce a task that allows multiple people to engage in a collaborative interaction with a standard robot arm.

\section{ROBOT ASSISTED TOWER CONSTRUCTION-A RESOURCE ALLOCATION TASK}

To enable the extension of current human robot collaboration research toward groups of people collaborating with a robot, we developed a novel resource allocation task that extends typical human-robot collaboration settings from dyadic to group settings.

We designed our task to meet several criteria: First, the task should enable data-collection about HRI and collaboration across a range of group sizes. That means the method should be flexible and allow the same data collection approach when studying a robot interacting with two, three, or more human collaborators. Second, the task should allow data collection of human-robot collaboration that employs a simple robot arm. Robot arms likely represent the most frequently deployed robots today, and robot arms are used across a broad range of human-robot collaboration contexts and studies (e.g., see Dragan et al. [2013], Huang et al. [2015], Nikolaidis et al. [2013], and Wilcox et al. [2013]). Further, using a simple robotic arm capable of manipulating physical objects will allow us to draw insights that cannot be gained from virtual agents and physically embodied robots that lack manipulation capabilities (e.g., Nao robot, Jibo, or iCat). Third, the task should allow data collection about human robot collaboration across multiple levels of analysis (individual, inter-personal, and groups). The method should allow the collection of process and outcome data related to the task and social dynamics. Fourth, the task should be applicable to different forms of robot control. That means it should be applicable to robots that are Wizard of Oz controlled or autonomously controlled and thus allow data collection that (1) aids empirical studies aimed at understanding human robot collaboration, (2) aids the technical development and evaluation of novel algorithmic collaboration approaches, and (3) aids the generative design of novel interaction approaches. Fifth, the task should enable research that aims at developing novel understanding about a robot's impact on groups as well as research that aims at developing novel technical solutions (e.g., distribution algorithms) that enable robots to function in groups.

To meet these criteria, we designed a task that places two or more human participants in a collaborative interaction with a robot. Drawing from established collaboration paradigms (e.g., see Goldberg and Maccoby [1965], Mitchell and Silver [1990], and Rosenbaum et al. [1980]), a tower construction task is used to engage participants in a collaborative interaction. However, deviating from established paradigms, the task not only introduces a robot but also a novel resource allocation component. Specifically, a robot is introduced that decides how resources are allocated among participants. The task is specified through a task description, a physical setup, and a specification of a simple robot operation.

\subsection{Task Description}

The task requires a group of human participants to build a structure as tall as possible out of building blocks in collaboration with a robot. The robot's task is to deliver resources (building blocks) to the team by picking them up from a stack located behind the robot and placing them in the front, where participants are located. The task of the human participants is to place the blocks to build the desired structure. The robot decides how to allocate blocks among participants. The task is completed once all blocks are placed. With these characteristics, the task models typical 
assembly or building tasks that are used in many other human-robot collaboration studies (e.g., see Hayes and Scassellati [2014, 2015], Khatib [1999], and Morioka and Sakakibara [2010]).

\subsection{Task Materials}

Task materials include a typical robot arm (e.g., Kuka YouBot) with a gripper as an end-effector, a set of building blocks, and a fixture to hold the blocks in place such that they can be reliably picked by the robot. The robot is placed on a table such that it can pick up building blocks from one side of the table and place them in front of the human collaborators on the other side of the table (Figure 3 ). Our example setup used for the case study utilizes 20 blocks that are stored in four stacks of five blocks each, but any number or configuration of blocks can be used. For the purposes of our case study, the number of blocks and stacks was chosen to meet several constraints: We wanted the number of blocks to be sufficiently high to allow groups to assemble a broad variety of tower designs and to allow for risky (in terms of height and stability) tower designs. We further wanted groups to be able to complete the task within 10 to 20 minutes. Finally, we wanted the blocks to be easily graspable and to remain in a fixed location so that the robot would not require object detection capabilities to grasp a block.

The task utilizes a standard robot arm for three reasons. First, robot arms are possibly the most frequently employed type of robots due to their reliability, precision, and strength. Since its invention [Scheinman 1969], their use has been growing rapidly with an estimated 1.5-1.75 million industrial robots in operation in the US, and projections see a three-fold increase in use by 2025 [Acemoglu and Restrepo 2017]. Second, advancements in safe HRI [Lasota et al. 2017] and humanrobot collaboration (e.g., see Hayes and Scassellati [2014, 2015], Khatib [1999], and Morioka and Sakakibara [2010]) have increasingly placed robot arms into direct collaborative interaction with people further extending its use and making it increasingly important to understand how such robots affect their social environment. Third, focusing on the use of a robotic arm that places resources directly extends current research approaches to human-robot collaboration, or teaming, the majority of which employ similar robot arms (e.g., see Hayes and Scassellati [2014b, 2015], Khatib [1999], and Morioka and Sakakibara [2010]).

\subsection{Robot Operation}

In the simplest application of the task, the robot relies only on simple pick and place operations to collaborate on the task. Pick-and-place is an "elementary" robot operation that almost any robot arm can perform and that most robot arms do perform in one way or another across many current application contexts. By focusing on such elementary robot operations or behaviors, the method aims to aid in collecting data from which it is possible to draw more generalizable conclusions than is possible from data generated from more complex robot behaviors.

\section{CASE STUDY: APPLYING THE TASK TO A STUDY THAT EXPLORES THE IMPACT OF RESOURCE DISTRIBUTION ON TEAMWORK OUTCOMES}

Whenever a robot collaborates with more than one human, it has to make decisions about the distribution of resources (e.g., social attention, task support, or physical resources). The robot has to decide to whom to attend, or whom to assist at any point in time. How such resources are distributed likely affects the social dynamics within the group with potential effects on immediate and long term team outcomes. For example, we know from prior work that people are uniquely attuned to inequalities and fairness in resource distribution (e.g., see Brosnan and de Waal [2014] and Lee [2018]), and even a simple change in a robot's distribution of gaze within a group of people can change participant roles [Mutlu et al. 2009]. Therefore, if we want to develop fundamental 
understanding about a robot's impact on group behavior and outcomes, it is important to understand whether and how resource distribution by a robot shapes group dynamics and outcomes.

To provide a case for the application of our task, we used it to explore the question of how a robot's distribution of resources affects team social dynamics and outcomes in a collaborative construction task. Specifically, we asked: Does the mere distribution of resources by a robot (equal vs. unequal between two human team members) affect team performance and perceptions of relationship quality?

\subsection{Experimental Design}

To explore our research question, we designed an exploratory, two-condition (equal vs. unequal distribution of resources), between participants study in which two human participants collaborate with a robot to complete a task-building a tower out of wooden blocks to be as high as possible. Our study directly extends current research on human-robot collaboration (e.g., see Hayes and Scassellati [2014b, 2015], Khatib [1999], and Morioka and Sakakibara [2010]) by adding a second human team member. Equality in resource distribution (Independent Variable) was operationalized through the robot's placement of 20 wooden building blocks required to complete a tower building task. In the equal distribution condition, the robot gives 10 blocks to each participant, making a $50 / 50$ distribution. In the unequal contribution condition, the robot gives the participant sitting in the right-hand side 13 blocks, and the participant on the left-hand 7 blocks, making a 65/35 distribution between the participants (Table 1). Although we controlled the robot remotely using a Wizard of $\mathrm{Oz}$ approach, we introduced the robot as autonomous to participants and lead them to believe that the robot was actively watching and listening to their actions during the tower building task in order for it to make block distribution decisions.

\subsection{Participants}

We recruited 124 participants $(\mathrm{N}=62$ teams) for this study using a combination of fliers and the university's online student recruitment system. Participants were provided compensation of either cash payments or student research credits. Of the 124 total participants recruited, 61.3 percent identified as female $(n=76), 37.9$ percent were male $(n=47)$, and one participant identified as non-binary. A plurality of participants identified their ethnicity as Asian (42.7\%, $\mathrm{n}=53$ ), followed by White $(33.9 \%, \mathrm{n}=42)$, Hispanic $(10.5 \%, \mathrm{n}=13)$, Other $(7.3 \%, \mathrm{n}=9)$, African American $(3.2 \%$, $\mathrm{n}=4)$, and Pacific Islander $(0.8 \%, \mathrm{n}=1)$, with two participants choosing not to identify their ethnicity. Ages ranged considerably from 18-66 years, but the median age of participants was 21 years. Twenty-four participants indicated that they knew their team partner before the study. Participants on average responded on a seven-point scale to be somewhat familiar with robots in general $(M=3.01, S D=1.5)$. Given this study occurred on a university campus and relied largely on an undergraduate and graduate participant pool, most participants were highly educated with over 75 percent $(n=94)$ reporting having at least some college education.

\subsection{Procedure}

The study procedures involved four steps totaling between 30 and 45 minutes.

Step 1-Pre Survey and Task Introduction: Participants first provided informed consent and filled out a pre-study survey that included demographic questions, questions about their familiarity with their study partner, and their familiarity with robots. Next, an experimenter led participants into a small laboratory where the robot was set up as described in the previous section. The experimenter asked participants to sit on one of the randomly assigned chairs.

Once seated, the experimenter told participants that the study involved testing an autonomous robot designed to help a two-person team build the tallest tower possible from a set of 20 wooden 
blocks. The experiment would consist of 20 rounds (one for each wooden block). At the start of each round, the robotic arm would give one of the participants a wooden block. That participant must use this block and place it to construct a tower in front of the robot. Once the block had been placed, the robotic arm would start the next round by picking up a new block and deciding to which team member to give it. This would continue for 20 rounds until all the blocks had been used. Participants were told that their goal was to build the tallest possible tower. They were free to talk and plan with one another, but they could only place a block on the tower if it was given to them by the robot (participants were explicitly told they could not share blocks with each other).

To highlight the interdependent nature of the task, we informed participants that each team could earn an extra team bonus of up to $\$ 10$ dependent on the height of the tower measured at the end of the building task. However, the team bonus would be paid out according to individual task contribution and split up according to the distribution of block placements. If a team earned a total bonus of $\$ 8$ and each participant placed 10 blocks on the tower, that would be a 50/50 split, and each participant would receive $\$ 4$. Thus, across conditions participants were told that the bonus compensation would depend on the team's success and that the bonus would be distributed according to the distribution of block placements. If the participants' tower fell down at anytime during the study, the table would be cleared, and the participants would continue building the tower with whatever blocks remained. They were reminded that this would significantly lower their bonus payment, as their tower would have fewer blocks. We used this reward design to model teamwork contexts in which both team success and individual contributions contribute to rewards and to highlight the interdependent nature of the collaboration task.

Step 2-Calibration Routine: The second step of the procedure was designed to create a believable experience of working with an autonomous robot. While the robot was operated by the experimenter through a remote operation interface (see Figure 4), it was introduced to participants as autonomous. The experimenter told participants that a machine learning approach had been used to train the robot on a dataset of hundreds of two person teams building similar wooden block towers. Based on this training, the robot would give a block to the person it thought at the moment could best help the team succeed toward building the highest tower.

To further emphasize the seemingly autonomous nature of the robot, and its seeming capability to sense the presence and behavior of the participants, we asked participants to complete a short calibration and training routine with the robot before starting the actual task. Participants were told that the robot has two main sensors, a Microsoft Kinect camera placed in front of the robot that is able to map and model the participants movements in 3D space, and a microphone. Both visual and audio information would be used by the robot to determine to whom it should give the next block. In actuality, none of the systems explained here were functional but were merely placed on the table. The first part of the calibration routine required each participant to pick up a wooden block three times with each hand in front of the robot. Participants were told that this data was used to build a 3D model of their movements. The second part of the calibration routine involved each participant uttering "Mary had a little lamb whose fleece was white as snow" three times in their normal speaking voice. Participants were told this was used to build a vocal model for the robot to understand their speech. In both cases, the experimenter looked at a laptop seemingly entering parameters and monitoring the progress of the calibration routine until it was completed in a satisfactory fashion.

Step 3-Building Task: After the completion of the calibration routine, the experimenter left the room telling participants that he/she would wait outside the room during the tower building activity. Instead, the experiment monitored a live video feed of the task in the nearby observation lab. 
Table 1. Order Robot Hands Out Blocks in Each Round for Each Condition

\begin{tabular}{|l|l|l|l|l|l|l|l|l|l|l|l|l|l|l|l|l|l|l|l|l|}
\hline Condition & 1 & 2 & 3 & 4 & 5 & 6 & 7 & 8 & 9 & 10 & 11 & 12 & 13 & 14 & 15 & 16 & 17 & 18 & 19 & 20 \\
\hline $65 / 35$ & $\mathrm{~A}$ & $\mathrm{~B}$ & $\mathrm{~A}$ & $\mathrm{~B}$ & $\mathrm{~A}$ & $\mathrm{~A}$ & $\mathrm{~A}$ & $\mathrm{~B}$ & $\mathrm{~A}$ & $\mathrm{~B}$ & $\mathrm{~A}$ & $\mathrm{~A}$ & $\mathrm{~A}$ & $\mathrm{~B}$ & $\mathrm{~A}$ & $\mathrm{~B}$ & $\mathrm{~A}$ & $\mathrm{~A}$ & $\mathrm{~A}$ & $\mathrm{~B}$ \\
\hline $50 / 50$ & $\mathrm{~A}$ & $\mathrm{~B}$ & $\mathrm{~A}$ & $\mathrm{~B}$ & $\mathrm{~A}$ & $\mathrm{~A}$ & $\mathrm{~B}$ & $\mathrm{~B}$ & $\mathrm{~A}$ & $\mathrm{~B}$ & $\mathrm{~A}$ & $\mathrm{~A}$ & $\mathrm{~B}$ & $\mathrm{~B}$ & $\mathrm{~A}$ & $\mathrm{~B}$ & $\mathrm{~A}$ & $\mathrm{~A}$ & $\mathrm{~B}$ & $\mathrm{~B}$ \\
\hline
\end{tabular}

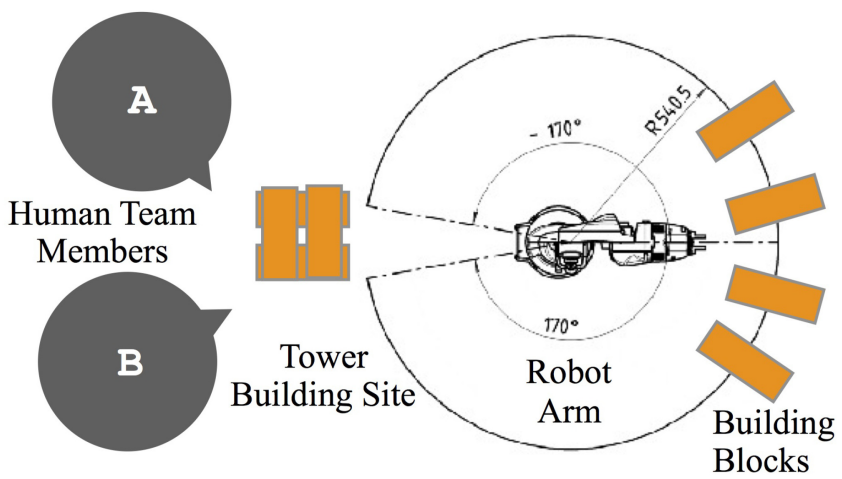

Fig. 3. Schematic overview of the human-robot collaboration setup with two human collaborators as used in our case study. The orange squares represent building blocks that are picked up by the robot from the stacks on the right and then delivered to the participants who are using them to assemble a structure.

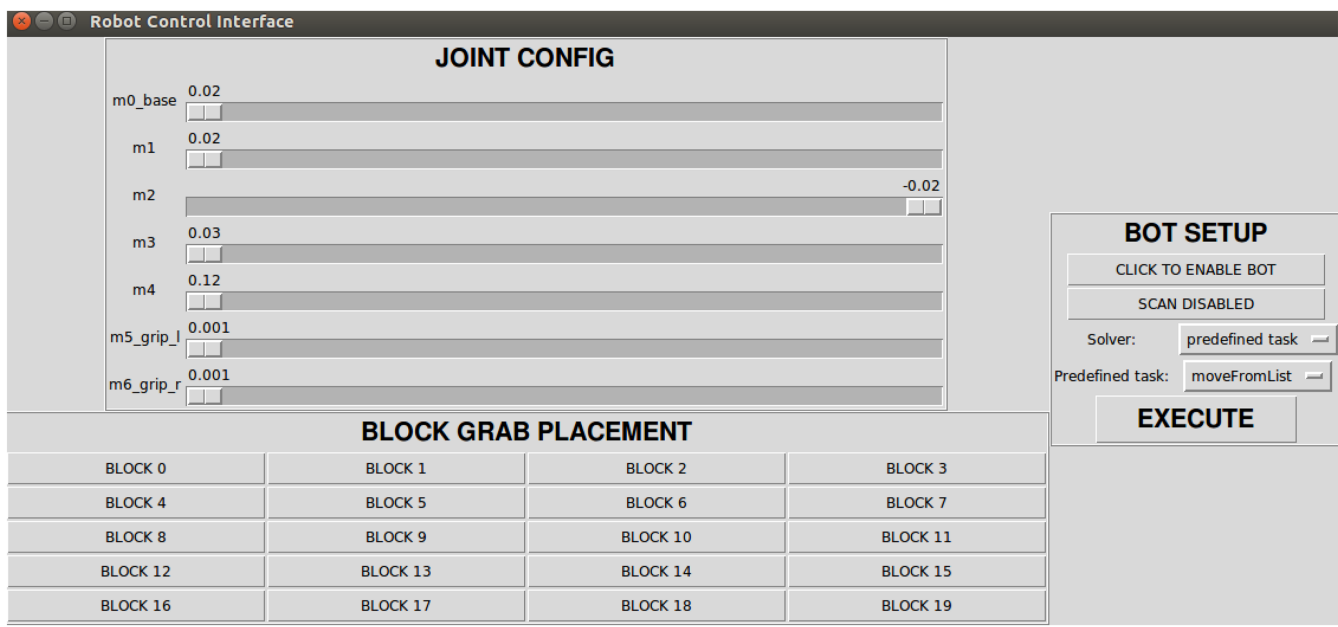

Fig. 4. Remote operator interface for Wizard of Oz control of the robot.

Participants were asked to start with the building task once the robot handed out the first block. Once a participant placed a block on the tower, the robot continued by passing out the next block. This continued until all 20 blocks were passed out to the participants. Based on the study condition, blocks were distributed in the sequences outlined in Table $1(\mathrm{~A}, \mathrm{~B}=$ participants $\mathrm{A}$ and $\mathrm{B}$ as in Figure 3).

At the completion of the task, the experimenter entered the room, measured the height of the tower with a ruler. The experimenter told all participants, irrespective of their actual performance, that their tower was in the 89th percentile of all towers measured so far and would thus receive $8 / 10$ points, equaling a team bonus of $\$ 8$. Based on the study condition and the distribution of 
blocks, participants were then ether paid both $\$ 4$ in the equal distribution (50/50) condition, or $\$ 6$ and $\$ 2$, respectively, in the unequal distribution (65/35) condition.

Step 4-Post-Survey and Debriefing: After receiving their reward payments, participants completed a series of survey measures including the subjective value inventory and open-ended questions asking them to reflect on the tower building activity and the robot's decision-making mechanism. Participants were then debriefed and told the true purpose of the study, that the robot was not actually watching or listen to them, and that the distribution they experienced was preprogrammed before they started the study.

\subsection{Measures}

To evaluate the impact of the robot's behavior on the team, we measured team performance and team members' satisfaction with the quality of the relationship with their team partner.

4.4.1 Team Performance. Our team performance measure was operationalized by collecting a measure of each team's tower height at the conclusion of the building task. An objective measure of overall team performance was analyzed both to establish whether distribution conditions significantly impacted task performance and, if so, to statistically control for the effects of these differences on how team members subjectively experience the task.

While team performance can be assessed in countless ways, researchers of group effectiveness or group performance largely agree that group performance can be assessed along three dimensions according to the degree to which "(1) the group's product meets or exceeds the needs of the users of that product; (2) group members interact in ways that allow the group to work more effectively over time, learning from each other and developing norms of operating that support high-quality performance; and (3) the group experience, on balance, satisfies rather than frustrates the needs of members [Hackman 1987]" ([Wageman 1995]; p. 148). Since, the latter two of the three performance criteria are more applicable to teams embedded in an organizational context over time, we decided to assess team performance only along the first dimension. We thus measured team performance by assessing the output according to the same criteria that team members received from the client (in this case, the experimenter): tower height of the final uncollapsed tower. The criterion to exclude blocks from an uncollapsed tower in the final score was included to emphasize the importance of the tower's overall structural integrity.

4.4.2 Relationship Satisfaction. We used slightly modified items from a four-item subscale (Feelings about Counterpart Relations) of the Subjective Value Inventory (SVI) from Curhan et al. [2006] to understand participant's satisfaction with their relationship with their team partner. Items were assessed on 7 -point Likert scales $(1=$ strongly disagree to $7=$ strongly agree $)$ and had high reliability $(\alpha=.82)$. The four items assessed the participants' general impression of their partner and the effect of the activity on their sense of trust and satisfaction ("The task made me trust my counterpart," "The task built a good foundation for a future relationship with my counterpart," "I am satisfied with the relationship with my counterpart as a result of this task," and "My counterpart made an overall good impression on me.").

\subsection{Results}

In order to test whether the robot, through mere differences in block allocation (equal vs. unequal distribution) was capable of influencing team member ratings of relationship satisfaction, a grouplevel relationship satisfaction score was calculated by averaging individual team member ratings within each team. Our approach to use an average at the team level is motivated both conceptually and empirically. Conceptually, we follow prior work that conceptualizes team relationship 
Table 2. Descriptive Statistics

\begin{tabular}{lrcccc}
\hline & Block Distribution & $\mathrm{N}$ & Mean & SD & SE \\
\hline Team Relationship Satisfaction & Unequal & 30 & 5.646 & 0.757 & 0.138 \\
Individual Relationship Satisfaction & Equal & 32 & 6.160 & 0.659 & 0.117 \\
& Unequal (Pos. A) & 30 & 5.578 & 0.774 & 0.136 \\
& Unequal (Pos. B) & 30 & 5.533 & 1.074 & 0.196 \\
& Equal (Pos. A) & 32 & 6.234 & 0.806 & 0.142 \\
Tower Height & Equal (Pos. B) & 32 & 6.094 & 0.800 & 0.141 \\
& Unequal & 30 & 11.483 & 4.841 & 0.884 \\
& Equal & 32 & 13.711 & 5.847 & 1.034 \\
\hline
\end{tabular}
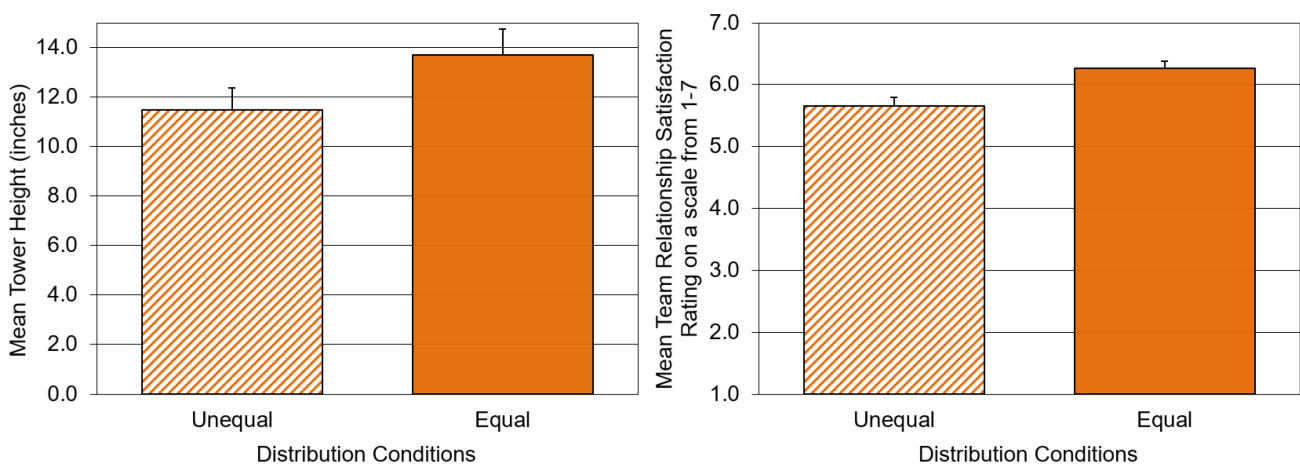

Fig. 5. Left: Mean tower height between distribution conditions. Not significant at the $p<.05$ level. Right: Mean ratings of team relationship satisfaction between distribution conditions. Significant at the $p<.01$ level. Error bars represent the standard error.

satisfaction and related constructs at the group level (e.g., see Beal et al. [2003]). Empirically, our data show that team members largely agree on the quality of the interpersonal relationship. Across both conditions, we find no significant difference in relationship satisfaction between group participants. Specifically, in the equal distribution condition, participants provided an average rating of relationship satisfaction of $M=6.1$ and $M=6.2$, a non-significant difference statistically; $t(1$, $62)=-0.7, \mathrm{p}=.49, \mathrm{~d}=-.18$. In the unequal distribution condition, participants provided an average rating of relationship satisfaction of $M=5.5$ and $M=5.8$, also a non-significant difference statistically; $\mathrm{t}(1,58)=-0.94, \mathrm{p}=.35, \mathrm{~d}=-.24$ (see Table 2 ).

We conducted a single independent samples t-test using the team relationship satisfaction score as the dependent variable. Levene's test of equality of variance was non-significant, indicating that equality of variance could be assumed, $\mathrm{F}(1,60)=1.78, \mathrm{p}=.19$. Results of the Student's $\mathrm{t}$ test demonstrate a significant difference between distribution conditions, $t(1,60)=-2.86, p=$ $.006, \mathrm{~d}=.73$, such that teams in the unequal distribution condition reported less team relationship satisfaction than those in the equal distribution condition (see Figure 4 and Table 3 ). Towers built by teams in the equal distribution condition were on average more than two inches higher than those in the unequal distribution condition. However, this difference was not statistically significant; $\mathrm{t}(1,60)=1.63, \mathrm{p}=.11$ (see Figure 5 and Table 3$)$.

\subsection{Case Study Discussion}

Results from this case study demonstrate the impact that a non-anthropomorphic robot can have on team dynamics and outcomes merely by the way it allocates resources among team members. 
Table 3. Independent Samples T-Test of Effects of Robot Block Distribution on Team Relationship Satisfaction

\begin{tabular}{lcccrrr}
\hline & & & & & \multicolumn{2}{c}{ 95\% CI for Cohen's d } \\
\cline { 6 - 8 } & $\mathrm{t}$ & $\mathrm{df}$ & $\mathrm{p}$ & Cohen's d & Lower & \multicolumn{1}{c}{ Upper } \\
Team Relationship Satisfaction & -2.857 & 60.00 & 0.006 & -0.726 & -1.238 & -0.209 \\
Tower Height & -1.628 & 60.00 & 0.109 & -0.414 & -0.916 & 0.092 \\
\hline
\end{tabular}

Specifically, a comparison of people's perceptions of their relationship with their partner across conditions revealed that team members in the unequal distribution condition reported a significantly more negative perception of their relationship than those in the equal distribution condition. Team relationship satisfaction was assessed after team members received the financial compensation dependent on the final block distribution. It is thus possible that the difference in relationship satisfaction is the result of a misattribution effect: Consistent with the mood as information theory [Schwarz and Clore 1983, 2003], participants receiving less might falsely attribute their negative reaction to the perception of their team member. Additionally, consistent with Staw's [1975] finding that people "use knowledge of performance as a cue by which they attribute characteristics to themselves, their work groups, and organizations," it is possible that participants receiving less money assumed that the relationship with their partner was impaired. In other words, performing worse, a participant might think that the team's bad dynamics were responsible for the outcome. However, inconsistent with these explanations, relationship satisfaction was not contingent upon the relative compensation people received as relationship satisfaction did not differ significantly between team members in the unequal distribution condition $t(1,58)=-0.94, \mathrm{p}=.35, \mathrm{~d}=-.24$. Additionally, relationship satisfaction dropped significantly between the equal and unequal conditions independent of whether a participant's compensation was higher (from $M=6.2$ to $M=$ $5.8 ; \mathrm{t}(1,60)=-2.34, \mathrm{p}=.02, \mathrm{~d}=-.6$ ) or lower (from $\mathrm{M}=6.1$ to $\mathrm{M}=5.5 ; \mathrm{t}(1,60)=-2.4, \mathrm{p}=.02$, $\mathrm{d}=-.6)$ than the teammate's, suggesting that relationship satisfaction was not determined by the compensation a participant received.

The case also provided initial evidence that sheds light onto the complex social role an industrial robot can take on when engaged in a group interaction. Table 4 shows a 19 second sequence from one of the teams participating in the study. One of the participants (left participant)) places her hand open on the table in expectation of receiving the next block. Then, seemingly unexpected to her, the robot places the block in front of the other participant (on the right). This creates a vulnerable moment for the participant on the left, who makes a tense facial expression and retracts her arm. Upon receiving the block, the participant on the right displays a nervous frown, seemingly embarrassed about the position the robot put him in. The sequence ends with with an exchange of gaze and affiliative smiles between participants as part of what seems an attempt to repair the awkward social situation the robot has placed them in. This exchange is interesting for several reasons: It demonstrates how a robot's simple pick-and-place behavior can become offensive as it places one participant in a vulnerable position through a seeming rejection. Further, the example demonstrates how a robot's simple behavior can impact interpersonal dynamics between multiple people. The awkward situation created by the robot makes a repair relevant. Finally, this example shows how even an industrial robot without any anthropomorphic physical features and without making a gesture that is specifically designed for social legibility (e.g., as in Dragan et al. [2013] and Takayama et al. [2011]) can elicit complex social dynamics within a group through one of the most basic robot operations an industrial robot can perform: picking up and placing an object. As such, this work builds on early findings on peoples' social relations to non-anthropomorphic 
Table 4. Short Interaction Sequence Evolving around a Robot's Block Placement

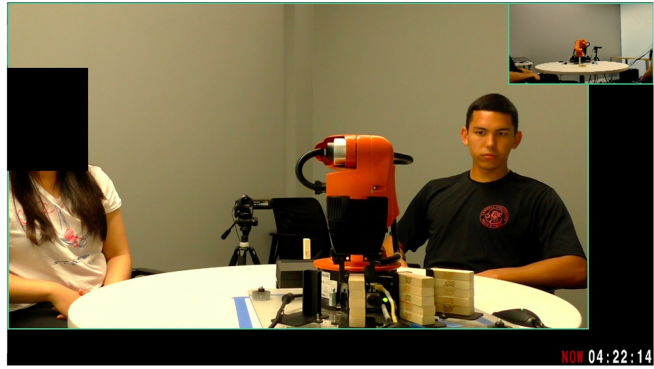

(1) 00 seconds: Participants are waiting for the robot to pick up the next block.

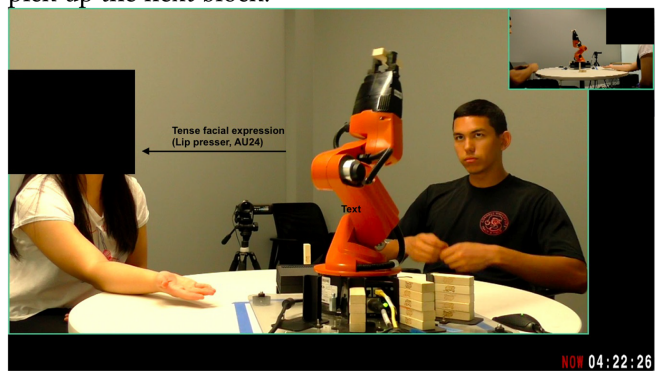

(3) 12 seconds: Upon realizing that the robot decided to give the next block to the participant on the right, the participant on the left makes a tense facial expression (lip presser, AU24), reflecting the vulnerable position the robot left her in.

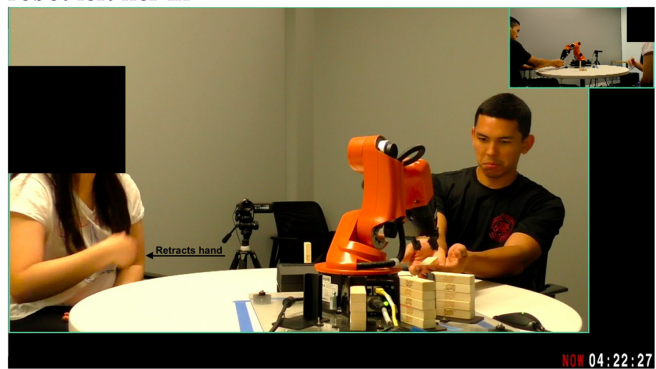

(5) 13 seconds: The participant on the left awkwardly retracts her hand as the other participant receives the block.

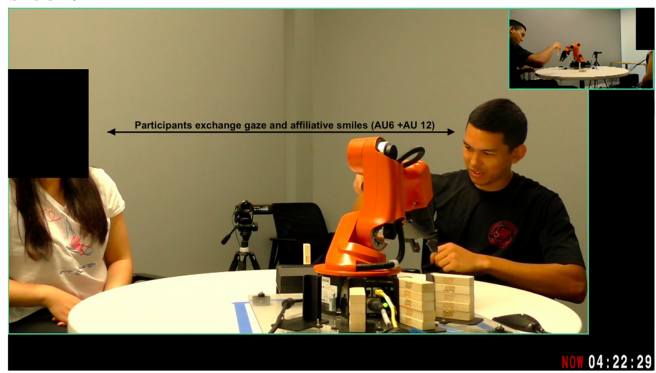

(7) 15 seconds: Both participants express affiliative smiles (lip corner puller, AU12, and cheek raiser, AU6).

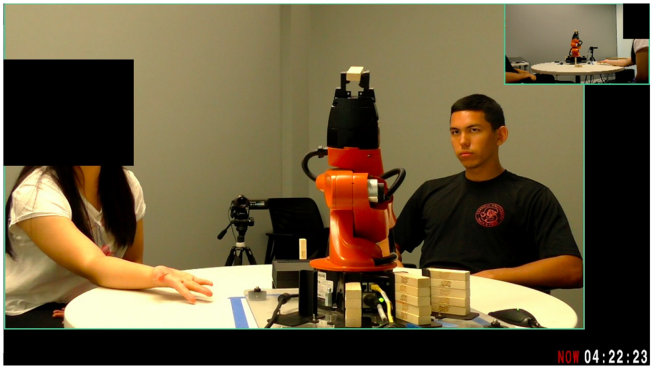

(2) 09 seconds: The participant on the left extends her arm in expectation of receiving the next block.

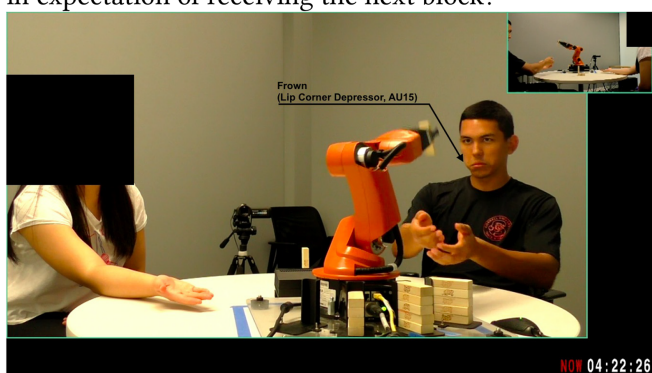

(4) 12 seconds: Upon receiving the next block, the participant on the right makes a tense expression of discomfort (lip corner depressor, AU15), which reflects the awkwardness of the situation the participants were placed in by the robot.

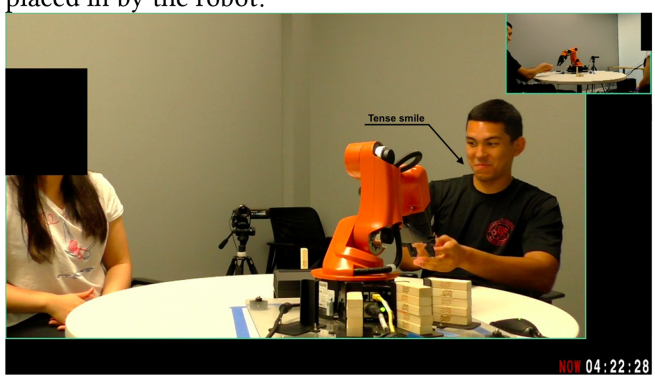

(6) 14 seconds: The participant on the right expresses an awkward smile toward the participant on the left in an attempt to repair the situation's awkwardness.

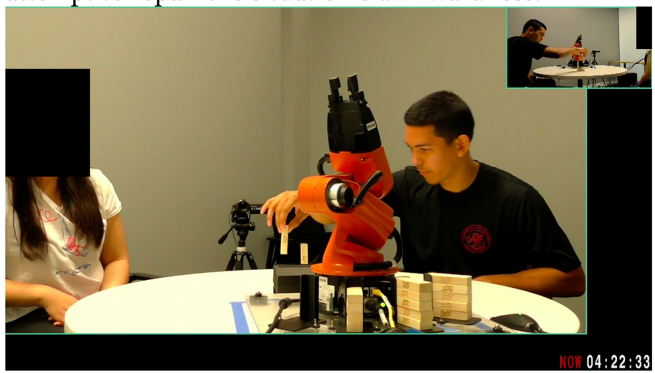

(8) 19 seconds: The participant on the right places the received block as the other participant is watching. 
robots (e.g., Forlizzi and DiSalvo [2006]) by showing that non-anthropomorphic robots can also shape peoples' social relations with each other in complex ways.

Our finding that the distribution of resources by a robot affects the team's overall relationship quality has implications for both design practice and theory. First, this study informs how we might design algorithms to assist groups of people. Our study demonstrates that the development of algorithms for robots that support teams in achieving optimal performance might face a difficult dilemma. On the one hand, a robot's assistance should help the team optimize overall task performance by distributing resources to the team member most capable of helping the team succeed. On the other hand, our study showed that distributing resources unequally can come at a social cost that might hurt team performance in the long term as studies of teams in organizations have highlighted the importance of relationship quality and cohesion for performance (e.g., see Cohen and Bailey [1997]). Thus, when designing algorithms for robots to assist teams, researchers face the challenge to find ways that optimize immediate task performance while also maintaining good social dynamics between members of the team.

Our finding that unequal resource distribution might lead to an impoverished interpersonal relationship perception has implications for our understanding of algorithmic resource distribution and fairness. Recent research on algorithmic resource distribution between multiple people has predominantly focused on understanding individuals' perceptions of the algorithm and its decisions (e.g., see Lee [2018] and Lee and Baykal [2017]). Less is known about how resource distribution and perception of those as fair or unfair affects peoples' interpersonal relationships with each other. Our study sheds light onto the possible negative interpersonal consequences that may arise from letting machines decide how to distribute resources.

Finally, our case study provided initial evidence about people's reasoning about a robot's decision-making in groups. We asked people about how they thought the robot makes resource distribution decisions, and we received a wide range of answers. For example, some participants thought resource distribution decisions depended on the robot's assessment of individual building skills: "I believe the robots actions were determined by looking at the way we placed the blocks in terms of location but as well as how we placed the blocks, like gentle, firm, or rigid. Because of my gentleness, I believe the robot chose me to place many of the blocks in order to prevent falls and build a higher tower." Other participants believed the robot's decisions were driven by participants' verbal utterances: "The robot seemed to judge it mainly at first based on what we said, since I stated [sic] not having done this before. But later on, it started taking into account our ideas such as different ways of stacking the blocks. I think it did a good job being responsive to our ideas." Thus, this task can help in collecting data about how people reason about a robot's decision-making in group situations and contribute to ongoing debates on the lay theories people form when interacting with algorithmic systems (e.g., see DeVito et al. [2018]).

\section{OVERALL DISCUSSION AND CONCLUSION}

We introduced Robot Assisted Tower Construction, a task that enables HRI researchers to collect data about human-robot collaboration and interaction that involves groups of people.

We demonstrated the utility of our approach through a case study that shows how this task enabled us to collect data on the impact of a robot's resource distribution through elementary pick and place behaviors on interpersonal dynamics and outcomes. Our work directly informs how research in HRI is carried out [Wobbrock 2012; Wobbrock and Kientz 2016] as it enables researchers to collect data about human robot collaboration and interaction that involves groups of people. More broadly, our article contributes to a growing effort of building HRI-specific research methodology (e.g., see Dautenhahn [2007], Bethel and Murphy [2010], Woods et al. [2006], Riek [2012], Steinfeld et al. [2006], and Hoffman and Ju [2014]). 


\subsection{Contributions with Respect to Existing Collaboration Tasks}

The resource distribution task is, of course, not the first task that engages a single robot in an interaction with multiple people. Other tasks exist, but they are are often difficult to replicate as they rely on highly complex study protocols that involve highly trained confederates (e.g., see Jung et al. [2015]), or complex games designed to explore one specific research question (e.g., see Oliveira et al. [2018], Short et al. [2017], and Strohkorb Sebo et al. [2018]), or a specific robot design (e.g., see Hoffman and Ju [2014]), or they focus on remotely operated rather than autonomous robots (e.g., see You and Robert [2017]).

The tower construction task has several characteristics that distinguish it in fundamental ways from existing tasks. First, existing collaboration tasks or groups designed to examine collaborations with anthropomorphic robots (e.g., a Nao robot). Tasks that rely on a standard robot arm have been exclusively designed for one human collaborator (e.g., see Hayes and Scassellati [2014, 2015], Khatib [1999], and Morioka and Sakakibara [2010]). The tower construction task contributes to HRI methods as it allows researchers to examine the social influence of a standard industrial robot arm. Second, existing human-robot group collaboration tasks are designed to study the impact of a robot's complex social behavior on groups such as conflict management behavior [Jung et al. 2015], expressions of vulnerability [Strohkorb Sebo et al. 2018], or social support behaviors [Oliveira et al. 2018]. The tower construction task contributes to HRI methods as it allows the examination of a robot's resource allocation behavior on group dynamics and outcomes. Understanding the impact of a robot's resource allocation behavior will be increasingly important as more and more robots are placed in group contexts that make it inevitable for a robot to decide about the allocation of resources. Third, the tower construction task contributes to HRI methods as it generates a context in which the mere pick and place behavior of a standard robot arm elicits changes in people's interpersonal interactions with each other. With the increased placement of robots in complex group context, it is important to understand not only how a robot's behavior shapes interactions with the robot but also among people with each other. Our task is uniquely suited to develop understanding about the latter.

A particular strength of this task is that it can be easily modified. For example, team size or team composition (e.g., by gender or culture) can be easily modified to study effects of a robot's collaborative behavior on group size and composition (see Figure 6). The task is constructed to allow a manipulation of group size without changing the basic procedures. Whether the robot allocates resources among two, three, or more people has no implications for the basic procedures of this task. Further, it is possible to study varying degrees of anthropomorphic embodiment, for example, by adding eyes to study gaze effects (e.g., as in Admoni et al. [2014] and Moon et al. [2014]), or to study different motion trajectories when handing over blocks (e.g., see Dragan et al. [2013]), or by adding more complex social interfaces through a screen as is common for many current human safe robot arms (e.g., iRobot's Baxter and Sawyer, ABB’s Yumi, or KUKA's LBR iiwa).

\subsection{Generalizability}

Our task allows the investigation of the impact of a robot's resource allocation behavior on the dynamics and outcomes of small work groups and teams. While the case study only employed groups of two participants each, it provides insights not only into a robot's influence on two-person groups but small groups work more generally for several reasons: First, while some researchers have questioned whether two people can constitute a group (e.g., see Moreland [2010]), others have argued that "although dyads can have properties that do not lend themselves to certain group process research (e.g., coalition formation) and have properties that can go beyond typical group processes (e.g., intimate relations and love), in most instances, groups of 

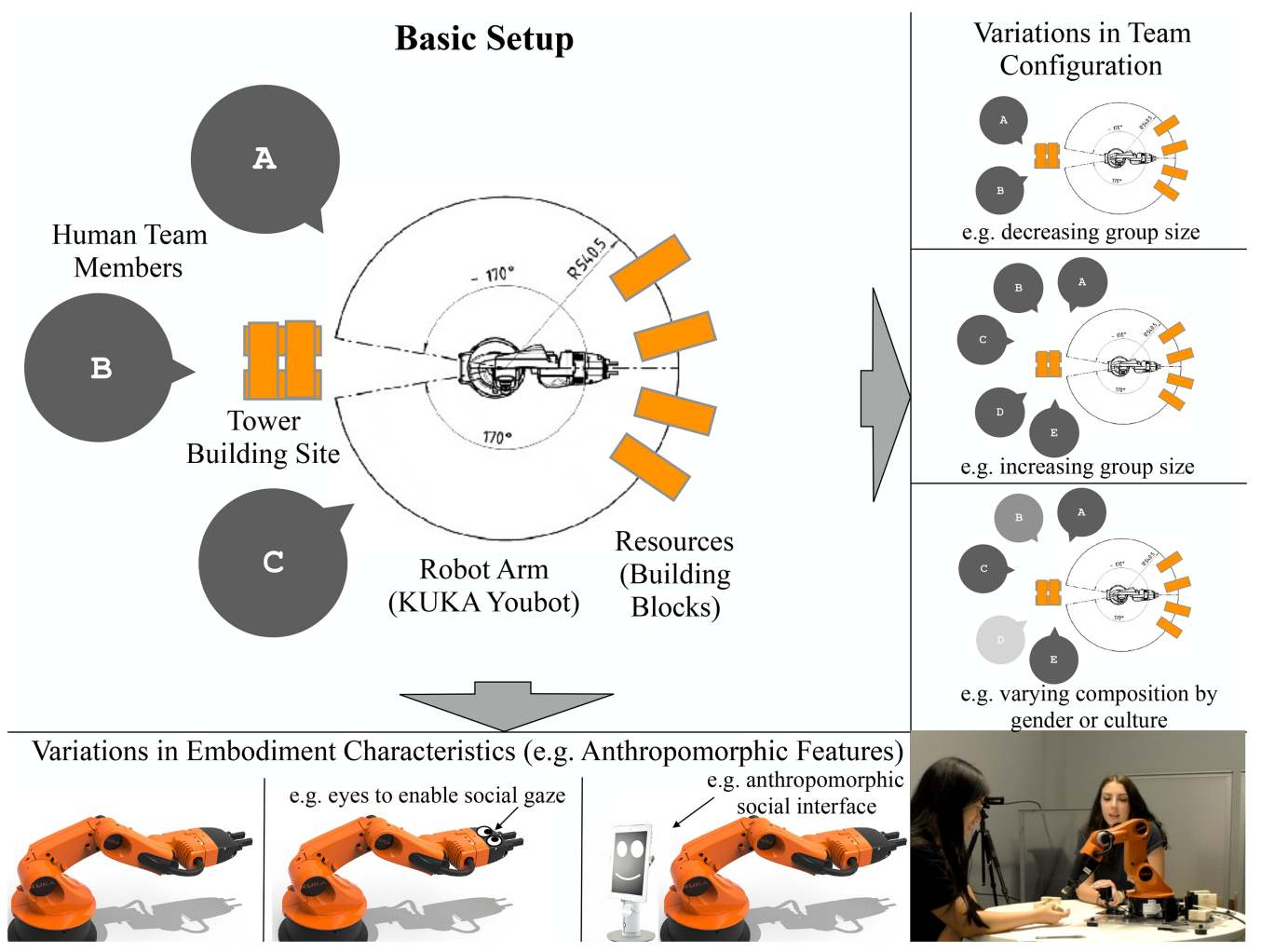

Fig. 6. Basic setup and possible alternate uses by modifying the task along dimensions of group size and composition as well as anthropomorphic design features of the robot.

two operate under the same principles and theories that explain group processes for groups of three and larger" [Williams 2010]. Therefore, while a case study with three human participants could have yielded additional insights about the robot's impact on group dynamics that are only observable in larger groups, we believe that our study provides evidence that the task can yield valuable data about a robot's impact on group dynamics more generally. Second, our collaboration task elicits group work that shares several criteria that scholars of small group work agree upon as being fundamental to group work (e.g., see Kozlowski and Bell [2003] and Cohen and Bailey [1997]): Members of work groups and teams (a) share one or more common goals, (b) are interdependent in their tasks (i.e., what one group member does influences another), and (c) interact socially. The tower construction tasks sets a shared goal of building the highest (or most stable or aesthetically pleasing) tower. Incentives, as in our case study, can be used to reinforced a shared goal. Group members are interdependent in their tasks as one member's block placement has direct implications for the other member's possible actions. Finally, the task encourages social interactions as group members deliberate about their actions. Third, a fundamental difference between the predominant form of human-robot collaboration teaming that involves a single robot and a single human, and human-robot and human-robot collaboration that involves a robot and a group of people is that, in the latter, interpersonal dynamics come into play. Our task constitutes the smallest possible setup that allows us to assess a robot's impact on interpersonal dynamics, and our case study indeed provided data that allows researchers to gain insight about such impact. 
Beyond studying how unequal resource distribution affects interpersonal dynamics and outcomes in small groups and teams, we believe that this task lends itself to a broad range of research questions in the area of human-robot collaboration. We envision the task to be used in the design and evaluation of novel algorithms for human robot collaboration in groups. For example, it could be used to evaluate resource distribution algorithms and test their impact on team performance and interpersonal dynamics. For example, a recent study by Claure and colleagues [2019] tested the impact of different resource allocation algorithms on team performance and trust. A second application context for this task could be as a scenario for designing novel interaction strategies for robots in group settings, using a generative Wizard of $\mathrm{Oz}$ paradigm (e.g., see Hoffman and Ju [2014]). For example, building on research that has shown how a robot's motion trajectories affect people's interpretation of a robot's behavior and intent [Dragan et al. 2013], the tower task can be used to perform elicitation studies that explore a wide range of motion trajectories (e.g., placing a block displaying hesitation vs. determination) and their impact on people. Third, the task lends itself to explore resource allocation as a deliberate intervention into a group's social dynamics. For example, building on other research that has shown that a robot can shift group dynamics by allocating increased attention to a less participating group member [Tennent et al. 2019], the robot could provide more resources to the person that is not actively contributing to the task or less resources to the person who dominates the floor in order to create more equal group participation. Finally, the tower construction task allows researchers to extend understanding of fairness in algorithmic decision-making by examining the interpersonal consequences of fairness in algorithmic decision-making. Existing research on algorithmic fairness has predominantly examined how decisions affect perceptions of the decision or the system making the decisions (e.g., see Lee and Baykal [2017]), but less is known about how the perception of an algorithmic decision as fair or unfair affects the perceptions people have of each other. Measuring perceptions of fairness will allow us to examine the possibility that the degree to which a robot's decision is perceived as fair or unfair explains its impact on interpersonal dynamics observed in the case study.

While the task to collaborate with a robot arm to construct a tower out of wooden blocks seems very specific, we believe this task can speak to a broad range of HRI scenarios. As we laid out in the introduction, questions regarding resource distribution emerge whenever robots are deployed in group settings. Our task provides a tool to examine questions surrounding a robot's resource distribution in group settings. Just as the Desert Survival Task [Lafferty et al. 1974] does not only speak to communication in a desert survival situation, and just as the famous ostracism task [Williams 1997] does not only speak to exclusion effects during a ball game, our task is intended to speak to any situation in which a robot distributes a resource among members of a group.

\subsection{Conclusion}

More than a century ago, German sociologist Georg Simmel reflected that adding a third member to a dyad brings with it a dramatic qualitative change as "the triad exhibits in its simplest form the sociological drama that informs all social life: the dialectic of freedom and constraint, of autonomy and heteronomy" [Coser 1971]. Adding just one more person to a dyadic human-robot collaboration brings with it complexities that are yet to be fully understood. We hope this task enables us to scale human-robot collaboration research beyond the dyad.

\section{ACKNOWLEDGMENT}

Any opinions, findings, and conclusions or recommendations expressed in this material are those of the authors and do not necessarily reflect the views of the National Science Foundation. We are thankful for the comments and valuable feedback we received from our reviewers. Special thanks also to Megan Zhou who helped with data collection and to our study participants. 


\section{REFERENCES}

Daron Acemoglu and Pascual Restrepo. 2020. Robots and jobs: Evidence from US labor markets. fournal of Political Economy 128, 6 (2020), 2188-2244.

Henny Admoni, Anca Dragan, Siddhartha S. Srinivasa, and Brian Scassellati. 2014. Deliberate delays during robot-to-human handovers improve compliance with gaze communication. In Proceedings of the 2014 ACM/IEEE International Conference on Human-Robot Interaction. ACM, 49-56.

Arash Ajoudani, Andrea Maria Zanchettin, Serena Ivaldi, Alin Albu-Schäffer, Kazuhiro Kosuge, and Oussama Khatib. 2018. Progress and prospects of the human-robot collaboration. Autonomous Robots (2018), 1-19.

Linda Argote and Paul S. Goodman. 1986. The organizational implications of robotics. In Implementing Advanced Technology, D. O. Davis (Ed.). Jossey-Bass, San Francisco, CA, 127-153.

Linda Argote, Paul S. Goodman, and David Schkade. 1983. The human side of robotics: How worker's react to a robot. In International Trends in Manufacturing Technology, T. H. Husband (Ed.). Springer-Verlag, New York, NY, 19-32.

Rajiv D. Banker, Joy M. Field, Roger G. Schroeder, and Kingshuk K. Sintia. 1996. Impact of work teams on manufacturing performance: A longitudinal field study. Academy of Management fournal 39, 4 (1996), 867-890.

Michael Barrett, Eivor Oborn, Wanda J. Orlikowski, and JoAnne Yates. 2012. Reconfiguring boundary relations: Robotic innovations in pharmacy work. Organization Science 23, 5 (2012), 1448-1466.

Andrea Bauer, Dirk Wollherr, and Martin Buss. 2008. Human-robot collaboration: A survey. International fournal of Humanoid Robotics 5, 1 (2008), 47-66.

Daniel J. Beal, Robin R. Cohen, Michael J. Burke, and Christy L. McLendon. 2003. Cohesion and performance in groups: A meta-analytic clarification of construct relations. Fournal of Applied Psychology 88, 6 (2003), 989.

Cindy L. Bethel and Robin R. Murphy. 2010. Review of human studies methods in HRI and recommendations. International fournal of Social Robotics 2, 4 (2010), 347-359.

Cynthia Breazeal, Nick DePalma, Jeff Orkin, Sonia Chernova, and Malte Jung. 2013. Crowdsourcing human-robot interaction: New methods and system evaluation in a public environment. Journal of Human-Robot Interaction 2, 1 (2013), 82-111.

Sarah F. Brosnan and Frans B. M. de Waal. 2014. Evolution of responses to (un) fairness. Science 346, 6207 (2014), 1251776.

Houston Claure, Yifang Chen, Jignesh Modi, Malte Jung, and Stefanos Nikolaidis. 2019. Reinforcement learning with fairness constraints for resource distribution in human-robot teams. arXiv preprint arXiv:1907.00313 (2019).

Susan G. Cohen and Diane E. Bailey. 1997. What makes teams work: Group effectiveness research from the shop floor to the executive suite. fournal of Management 23, 3 (1997), 239-290.

Filipa Correia, Samuel Mascarenhas, Rui Prada, Francisco S. Melo, and Ana Paiva. 2018. Group-based emotions in teams of humans and robots. In Proceedings of the 2018 ACM/IEEE International Conference on Human-Robot Interaction. ACM, 261-269.

Lewis A. Coser. 1971. Masters of Sociological Thought: Ideas in Historical and Social Context. Houghton Mifflin Harcourt P.

Jared R. Curhan, Hillary Anger Elfenbein, and Heng Xu. 2006. What do people value when they negotiate? Mapping the domain of subjective value in negotiation. Journal of Personality and Social Psychology 91, 3 (2006), 493.

Kerstin Dautenhahn. 2007. Methodology \& themes of human-robot interaction: A growing research field. International fournal of Advanced Robotic Systems 4, 1 (2007), 15.

Michael A. DeVito, Jeffrey T. Hancock, Megan French, Jeremy Birnholtz, Judd Antin, Karrie Karahalios, Stephanie Tong, and Irina Shklovski. 2018. The algorithm and the user: How can HCI use lay understandings of algorithmic systems? In Extended Abstracts of the 2018 CHI Conference on Human Factors in Computing Systems. ACM, panel04.

Lorin Dole. 2017. The influence of a robotâs mere presence on human communication. Ph.D. Dissertation (2017).

Anca D. Dragan, Kenton C. T. Lee, and Siddhartha S. Srinivasa. 2013. Legibility and predictability of robot motion. In Proceedings of the 8th ACM/IEEE International Conference on Human-Robot Interaction. IEEE Press, 301-308.

Jean-Pierre Durand, Paul Stewart, and Juan José Castillo. 1999. Teamwork in the Automobile Industry: Radical Change or Passing Fashion? Springer.

Jodi Forlizzi and Carl DiSalvo. 2006. Service robots in the domestic environment: A study of the Roomba vacuum in the home. In Proceedings of the 1st ACM SIGCHI/SIGART Conference on Human-Robot Interaction. ACM, 258-265.

Moses H. Goldberg and Eleanor E. Maccoby. 1965. Children's acquisition of skill in performing a group task under two conditions group formation. Journal of Personality and Social Psychology 2, 6 (1965), 898.

J. Hackman. 1987. R, 1987, The design of work teams. Handbook of Organizational Behavior (1987), 315-342.

J. Richard Hackman and Charles G. Morris. 1975. Group tasks, group interaction process, and group performance effectiveness: A review and proposed integration. In Advances in Experimental Social Psychology. Vol. 8. Elsevier, 45-99.

Bradley Hayes and Brian Scassellati. 2014. Online development of assistive robot behaviors for collaborative manipulation and human-robot teamwork. In Proceedings of the Machine Learning for Interactive Systems (MLIS) Workshop at AAAI.

Bradley Hayes and Brian Scassellati. 2015. Effective robot teammate behaviors for supporting sequential manipulation tasks. In IEEE/RSf International Conference on Intelligent Robots and Systems (IROS'15). IEEE, 6374-6380. 
Bradley Hayes and Brian Scassellati. 2016. Autonomously constructing hierarchical task networks for planning and humanrobot collaboration. In Proceedings of the IEEE International Conference on Robotics and Automation (ICRA'16). IEEE, 5469-5476.

Pamela J. Hinds, Teresa L. Roberts, and Hank Jones. 2004. Whose job is it anyway? A study of human-robot interaction in a collaborative task. Human-Computer Interaction 19, 1 (2004), 151-181.

Guy Hoffman and Cynthia Breazeal. 2007. Effects of anticipatory action on human-robot teamwork efficiency, fluency, and perception of team. In Proceedings of the ACM/IEEE International Conference on Human-Robot Interaction. ACM, 1-8.

Guy Hoffman and Wendy Ju. 2014. Designing robots with movement in mind. Journal of Human-Robot Interaction 3, 1 (2014), 91-122.

Guy Hoffman, Oren Zuckerman, Gilad Hirschberger, Michal Luria, and Tal Shani Sherman. 2015. Design and evaluation of a peripheral robotic conversation companion. In Proceedings of the 10th Annual ACM/IEEE International Conference on Human-Robot Interaction. ACM, 3-10.

Chien-Ming Huang, Maya Cakmak, and Bilge Mutlu. 2015. Adaptive coordination strategies for human-robot handovers. In Robotics: Science and Systems.

Tariq Iqbal, Samantha Rack, and Laurel D. Riek. 2016. Movement coordination in human-robot teams: A dynamical systems approach. IEEE Transactions on Robotics 32, 4 (2016), 909-919.

Tariq Iqbal and Laurel D. Riek. 2017. Coordination dynamics in multihuman multirobot teams. IEEE Robotics and Automation Letters 2, 3 (2017), 1712-1717.

Malte Jung, Jan Chong, and Larry Leifer. 2012. Group hedonic balance and pair programming performance: Affective interaction dynamics as indicators of performance. In Proceedings of the SIGCHI Conference on Human Factors in Computing Systems. ACM, 829-838.

Malte F. Jung. 2016. Coupling interactions and performance: Predicting team performance from thin slices of conflict. ACM Transactions on Computer-Human Interaction (TOCHI) 23, 3 (2016), 18.

Malte F. Jung, Jin Joo Lee, Nick DePalma, Sigurdur O. Adalgeirsson, Pamela J. Hinds, and Cynthia Breazeal. 2013. Engaging robots: Easing complex human-robot teamwork using backchanneling. In Proceedings of the 2013 Conference on Computer Supported Cooperative Work. ACM, 1555-1566.

Malte F. Jung, Nikolas Martelaro, and Pamela J. Hinds. 2015. Using robots to moderate team conflict: The case of repairing violations. In Proceedings of the 10th Annual ACM/IEEE International Conference on Human-Robot Interaction. ACM, 229-236.

Takayuki Kanda, Masahiro Shiomi, Zenta Miyashita, Hiroshi Ishiguro, and Norihiro Hagita. 2010. A communication robot in a shopping mall. IEEE Transactions on Robotics 26, 5 (2010), 897-913.

Oussama Khatib. 1999. Mobile manipulation: The robotic assistant. Robotics and Autonomous Systems 26, 2-3 (1999), 175183.

Ross A. Knepper, Christoforos I. Mavrogiannis, Julia Proft, and Claire Liang. 2017. Implicit communication in a joint action. In Proceedings of the 2017 ACM/IEEE International Conference on Human-Robot Interaction. ACM, 283-292.

Steve W. J. Kozlowski and Bradford S. Bell. 2003. Work groups and teams in organizations. Handbook of Psychology (2003), 333-375.

J. C. Lafferty, Patrick M. Eady, and J. Elmers. 1974. The desert survival problem. Experimental Learning Methods (1974).

Harvey J. Langholtz, Antoinette T. Marty, Christopher T. Ball, and Eric C. Nolan. 2002. Resource-Allocation Behavior. Springer Science \& Business Media.

Przemyslaw A. Lasota, Terrence Fong, Julie A. Shah, et al. 2017. A survey of methods for safe human-robot interaction. Foundations and Trends ${ }^{\circledR}$ in Robotics 5, 4 (2017), 261-349.

Min Kyung Lee. 2018. Understanding perception of algorithmic decisions: Fairness, trust, and emotion in response to algorithmic management. Big Data \& Society 5, 1 (2018), 2053951718756684.

Min Kyung Lee and Su Baykal. 2017. Algorithmic mediation in group decisions: Fairness perceptions of algorithmically mediated vs. discussion-based social division. In CSCW. 1035-1048.

Min Kyung Lee, Sara Kiesler, Jodi Forlizzi, and Paul Rybski. 2012. Ripple effects of an embedded social agent: A field study of a social robot in the workplace. In Proceedings of the SIGCHI Conference on Human Factors in Computing Systems. ACM, 695-704.

Yosuke Matsusaka, Shinya Fujie, and Tetsunori Kobayashi. 2001. Modeling of conversational strategy for the robot participating in the group conversation. In Proceedings of the 7th European Conference on Speech Communication and Technology.

Yoichi Matsuyama, Iwao Akiba, Shinya Fujie, and Tetsunori Kobayashi. 2015. Four-participant group conversation: A facilitation robot controlling engagement density as the fourth participant. Computer Speech \& Language 33, 1 (2015), $1-24$.

Joseph Edward McGrath. 1984. Groups: Interaction and Performance. Vol. 14. Prentice-Hall Englewood Cliffs, NJ. 
Joseph E. McGrath. 1991. Time, interaction, and performance (TIP): A theory of groups. Small Group Research 22, 2 (1991), 147-174.

Terence R. Mitchell and William S. Silver. 1990. Individual and group goals when workers are interdependent: Effects on task strategies and performance. fournal of Applied Psychology 75, 2 (1990), 185.

AJung Moon, Daniel M. Troniak, Brian Gleeson, Matthew K. X. J. Pan, Minhua Zheng, Benjamin A. Blumer, Karon MacLean, and Elizabeth A. Croft. 2014. Meet me where I'm gazing: How shared attention gaze affects human-robot handover timing. In Proceedings of the 2014 ACM/IEEE International Conference on Human-Robot Interaction. ACM, 334-341.

Richard L. Moreland. 2010. Are dyads really groups? Small Group Research 41, 2 (2010), 251-267.

Masashiro Morioka and Shinsuke Sakakibara. 2010. A new cell production assembly system with human-robot cooperation. CIRP Annals 59, 1 (2010), 9-12.

Bilge Mutlu and Jodi Forlizzi. 2008. Robots in organizations: The role of workflow, social, and environmental factors in human-robot interaction. In Proceedings of the 3rd ACM/IEEE International Conference on Human Robot Interaction. ACM, 287-294.

Bilge Mutlu, Toshiyuki Shiwa, Takayuki Kanda, Hiroshi Ishiguro, and Norihiro Hagita. 2009. Footing in human-robot conversations: How robots might shape participant roles using gaze cues. In Proceedings of the 4th ACM/IEEE International Conference on Human Robot Interaction. ACM, 61-68.

Stefanos Nikolaidis, Przemyslaw Lasota, Gregory Rossano, Carlos Martinez, Thomas Fuhlbrigge, and Julie Shah. 2013. Human-robot collaboration in manufacturing: Quantitative evaluation of predictable, convergent joint action. In Proceedings of the 44th International Symposium on Robotics (ISR'13). IEEE, 1-6.

Taiichi Ohno. 1988. Toyota Production System: Beyond Large-Scale Production. CRC Press.

Raquel Oliveira, Patrícia Arriaga, Patrícia Alves-Oliveira, Filipa Correia, Sofia Petisca, and Ana Paiva. 2018. Friends or foes?: Socioemotional support and gaze behaviors in mixed groups of humans and robots. In Proceedings of the 2018 ACM/IEEE International Conference on Human-Robot Interaction. ACM, 279-288.

Raquel Oliveira, Patricia Arriaga, and Ana Paiva. 2019. Future trends in research methodologies for human-robot interactions in groups. (2019).

Byron Reeves and Clifford Ivar Nass. 1996. The Media Equation: How People Treat Computers, Television, and New Media Like Real People and Places. Cambridge University Press.

Laurel D. Riek. 2012. Wizard of Oz studies in HRI: A systematic review and new reporting guidelines. fournal of HumanRobot Interaction 1, 1 (2012), 119-136.

Nina Riether, Frank Hegel, Britta Wrede, and Gernot Horstmann. 2012. Social facilitation with social robots? In Proceedings of the 7th Annual ACM/IEEE International Conference on Human-Robot Interaction. ACM, 41-48.

Milton E. Rosenbaum, Danny L. Moore, John L. Cotton, Michael S. Cook, Rex A. Hieser, M. Nicki Shovar, and Morris J. Gray. 1980. Group productivity and process: Pure and mixed reward structures and task interdependence. Fournal of Personality and Social Psychology 39, 4 (1980), 626.

Allison Sauppé and Bilge Mutlu. 2015. The social impact of a robot co-worker in industrial settings. In Proceedings of the 33rd Annual ACM Conference on Human Factors in Computing Systems. ACM, 3613-3622.

Brian Scassellati, Henny Admoni, and Maja Matarić. 2012. Robots for use in autism research. Annual Review of Biomedical Engineering 14 (2012), 275-294.

Victor David Scheinman. 1969. Design of a Computer Controlled Manipulator. Technical Report. Stanford Univ. Calif. Dept of Computer Science.

Matthias Scheutz, Paul Schermerhorn, and James Kramer. 2006. The utility of affect expression in natural language interactions in joint human-robot tasks. In Proceedings of the 1st ACM SIGCHI/SIGART Conference on Human-Robot Interaction. ACM, 226-233.

Norbert Schwarz and Gerald L. Clore. 1983. Mood, misattribution, and judgments of well-being: Informative and directive functions of affective states. Journal of Personality and Social Psychology 45, 3 (1983), 513.

Norbert Schwarz and Gerald L. Clore. 2003. Mood as information: 20 years later. Psychological Inquiry 14, 3-4 (2003), 296303.

Julie Shah, James Wiken, Brian Williams, and Cynthia Breazeal. 2011. Improved human-robot team performance using Chaski, a human-inspired plan execution system. In Proceedings of the 6th International Conference on Human-robot Interaction. ACM, 29-36.

Julie A. Shah. 2017. Enhancing human capability with intelligent machine teammates. In Proceedings of the 16th Conference on Autonomous Agents and MultiAgent Systems. International Foundation for Autonomous Agents and Multiagent Systems, 4-4.

Solace Shen, Petr Slovak, and Malte F. Jung. 2018. Stop. I see a conflict happening.: A robot mediator for young children's interpersonal conflict resolution. In Proceedings of the 2018 ACM/IEEE International Conference on Human-Robot Interaction. ACM, 69-77. 
Elaine Schaertl Short, Katelyn Swift-Spong, Hyunju Shim, Kristi M. Wisniewski, Deanah Kim Zak, Shinyi Wu, Elizabeth Zelinski, and Maja J. Matarić. 2017. Understanding social interactions with socially assistive robotics in intergenerational family groups. In Proceedings of the 26th IEEE International Symposium on Robot and Human Interactive Communication (RO-MAN'17). IEEE, 236-241.

Rosanne M. Siino and Pamela J. Hinds. 2005. Robots, gender \& sensemaking: Sex segregation's impact on workers making sense of a mobile autonomous robot. In Proceedings of the 2005 IEEE International Conference on Robotics and Automation (ICRA'05). IEEE, 2773-2778.

Garold Stasser and William Titus. 1985. Pooling of unshared information in group decision making: Biased information sampling during discussion. Journal of Personality and Social Psychology 48, 6 (1985), 1467.

Barry M. Staw. 1975. Attribution of the âcausesâ of performance: A general alternative interpretation of cross-sectional research on organizations. Organizational Behavior and Human Performance 13, 3 (1975), 414-432.

Aaron Steinfeld, Terrence Fong, David Kaber, Michael Lewis, Jean Scholtz, Alan Schultz, and Michael Goodrich. 2006. Common metrics for human-robot interaction. In Proceedings of the 1st ACM SIGCHI/SIGART Conference on HumanRobot Interaction. ACM, 33-40.

Sarah Strohkorb Sebo, Margaret Traeger, Malte Jung, and Brian Scassellati. 2018. The ripple effects of vulnerability: The effects of a robot's vulnerable behavior on trust in human-robot teams. In Proceedings of the 2018 ACM/IEEE International Conference on Human-Robot Interaction. ACM, 178-186.

Kristen Stubbs, Pamela J. Hinds, and David Wettergreen. 2007. Autonomy and common ground in human-robot interaction: A field study. IEEE Intelligent Systems 22, 2 (2007).

Leila Takayama, Doug Dooley, and Wendy Ju. 2011. Expressing thought: Improving robot readability with animation principles. In Proceedings of the 6th ACM/IEEE International Conference on Human-Robot Interaction (HRI'11). IEEE, 69-76.

Hamish Tennent, Solace Shen, and Malte Jung. 2019. Micbot: A peripheral robotic object to shape conversational dynamics and team performance. In Proceedings of the 2019 14th ACM/IEEE International Conference on Human-Robot Interaction (HRI). IEEE, 133-142.

Steven P. Vallas. 2003. Why teamwork fails: Obstacles to workplace change in four manufacturing plants. American Sociological Review 68, 2 (2003), 223.

Marynel Vázquez, Elizabeth J. Carter, Braden McDorman, Jodi Forlizzi, Aaron Steinfeld, and Scott E. Hudson. 2017. Towards robot autonomy in group conversations: Understanding the effects of body orientation and gaze. In Proceedings of the 2017 ACM/IEEE International Conference on Human-Robot Interaction. ACM, 42-52.

Janet Vertesi. 2015. Seeing Like a Rover: How Robots, Teams, and Images Craft Knowledge of Mars. University of Chicago Press.

Ruth Wageman. 1995. Interdependence and group effectiveness. Administrative Science Quarterly (1995), 145-180.

Ronald Wilcox, Stefanos Nikolaidis, and Julie Shah. 2012. Optimization of temporal dynamics for adaptive human-robot interaction in assembly manufacturing. In Proceedings of the Robotics Science and Systems. 441-448.

Kipling D. Williams. 1997. Social ostracism. In Aversive Interpersonal Behaviors. Springer, 133-170.

Kipling D. Williams. 2010. Dyads can be groups (and often are). Small Group Research 41, 2 (2010), 268-274.

Jacob O. Wobbrock. 2012. Seven research contributions in HCI. Studies 1, 1 (2012), 52-80.

Jacob O. Wobbrock and Julie A. Kientz. 2016. Research contributions in human-computer interaction. Interactions 23, 3 (2016), 38-44.

S. Woods, Michael L.Walters, Kheng Lee Koay, and Kerstin Dautenhahn. 2006. Methodological issues in HRI: A comparison of live and video-based methods in robot to human approach direction trials. In Proceedings of the 15th IEEE International Symposium on Robot and Human Interactive Communication, ROMAN.

Anita Williams Woolley, Ishani Aggarwal, and Thomas W. Malone. 2015. Collective intelligence and group performance. Current Directions in Psychological Science 24, 6 (2015), 420-424.

Stefan Wuchty, Benjamin F. Jones, and Brian Uzzi. 2007. The increasing dominance of teams in production of knowledge. Science 316, 5827 (2007), 1036-1039.

Sangseok You and Lionel Robert. 2017. Emotional attachment, performance, and viability in teams collaborating with embodied physical action (EPA) robots. AIS.

Received March 2019; revised December 2019; accepted April 2020 